\title{
High-resolution melting analysis coupled with next-generation sequencing as a simple tool for the identification of a novel somatic BRCA2 variant: a case report
}

\author{
Alessandra Costella ${ }^{1}$, Rossella De Leo ${ }^{2}$, Donatella Guarino ${ }^{1}$, Marco D'Indinosante ${ }^{2}$, Paola Concolino ${ }^{1,3}$, \\ Giorgia Mazzuccato', Andrea Urbani 1,3,4, Giovanni Scambia², Ettore Capoluongo 3,5, \\ Anna Fagotti ${ }^{2}$ and Angelo Minucci, ${ }^{1,3}$
}

\begin{abstract}
In a 72-year-old woman with no associated personal or family history of breast and/or ovarian cancers, we identified a novel somatic pathogenic BRCA2 variant (c.18_28delAGAGAGGCCAA, p.Lys6Asnfs*4) using next-generation sequencing (NGS). The variant allele frequency (VAF) was $16 \%$, and Sanger sequencing was unable to identify this variant. Adopting a high-resolution melting analysis strategy coupled with NGS, we successfully highlighted the presence of the c.18_28delAGAGAGGCCAA allele.
\end{abstract}

Keywords: High-resolution melting analysis; next-generation sequencing; novel somatic BRCA2 variant

Testing BRCA1/2 (BRCA) genes on formalin-fixed paraffin-embedded (FFPE) or fresh tissue (FT) samples permits the simultaneous assessment of both somatic and germline variants using an easily-accessible material that is routinely available in any pathology laboratory worldwide. FFPE and FT samples are histologically heterogeneous ${ }^{1}$, while tumor-specific DNA contains varying proportions of contaminating DNA from normal cells.

Next-generation sequencing (NGS) methods have the potential to detect variants at low admixture levels, offering a potential solution to this challenging type of analysis $^{2}$. Because of the poor quality of extracted DNA and to a low sequencing signal, variants in DNA from FFPE and FT sources are difficult to confirm using Sanger

Correspondence: Angelo Minucci (angelo.minucci@virgilio.it)

(angelo.minucci@policlinicogemelli.it)

${ }^{1}$ Area Diagnostica di Laboratorio IRCCS, Rome, Italy

${ }^{2}$ Department of Obstetrics and Gynecology, Division of Gynecologic Oncology, Rome, Italy

Full list of author information is available at the end of the article sequencing. Furthermore, these types of sources cannot be re-analyzed by NGS because of the small amount of DNA. To avoid considering these variants to be PCR artefacts, it is highly recommended to use alternative methodologies.

In this context, we used high-resolution melting analysis (HRMA) as a simple, cost-effective, rapid and sensitive method to confirm a novel somatic $B R C A 2$ variant (c.18_28delAGAGAGGCCAA, p.Lys6Asnfs*4) that was previously identified by NGS in a patient with high-grade serous ovarian cancer (HGSOC).

The present study involved a 72-year-old woman who presented to an oncologist with a complex right ovarian mass and elevated CA-125 level. Her gynecological history was negative. A transvaginal and transabdominal ultrasound examination revealed a multilocular solid cyst with $>10$ locules, papillary projections, and irregular surface with a Color Score of 4 . Computed tomography of her abdomen and pelvis showed a $10 \times 5 \mathrm{~cm}$ right ovarian mass and diffuse peritoneal enhancement, consistent with

\section{(c) The Author(s) 2018}

(c) (i) Open Access This article is licensed under a Creative Commons Attribution 4.0 International License, which permits use, sharing, adaptation, distribution and reproduction cc) in any medium or format, as long as you give appropriate credit to the original author(s) and the source, provide a link to the Creative Commons license, and indicate if changes were made. The images or other third party material in this article are included in the article's Creative Commons license, unless indicated otherwise in a credit line to the material. If material is not included in the article's Creative Commons license and your intended use is not permitted by statutory regulation or exceeds the permitted use, you will need to obtain permission directly from the copyright holder. To view a copy of this license, visit http://creativecommons.org/licenses/by/4.0/. 
peritoneal carcinomatosis. Ovarian cancer was suspected, and the patient consented to complete surgical staging. She underwent a total abdominal hysterectomy, bilateral salpingo-oophorectomy, partial pelvic peritonectomy, and radical omentectomy 2 months after her initial presentation. The surgery was largely uncomplicated, with no significant hemostasis or coagulation issues, and optimal cytoreduction was achieved. Surgery was followed by six cycles of chemotherapy with paclitaxel and carboplatin. Written informed consent was obtained to allow $B R C A$ testing to be performed after the pathological diagnosis of HGSOC was made.

DNA was extracted from FT HGSOC sections from areas with a minimum neoplastic cellularity of $70 \%$ using the MagCore Genomic DNATissue Kit by MagCore HF16 Plus (Diatech Lab Line, Jesi, Italy). The DNA concentration and quality were determined using a Qubit dsDNA HS assay (Thermo Fisher Scientific, Waltham, MA, USA).
$B R C A$ analysis was performed using the Devyser BRCA kit (Devyser, Hägersten, Sweden). Sequencing reactions were carried out on the MiSeq instrument (Illumina, CA, USA). NGS data were processed using the Amplicon Suite software (SmartSeq s.r.l., Novara, Italy) with the parameters of aligning reads to the HG19 reference genome and to generate run metrics, including the depth of sequencing, total read count, and quality. In addition, $B R C A$ large genomic rearrangements were also investigated as previously reported ${ }^{3,4}$.

Sanger sequencing and PCR-HRMA were performed on an ABI 3500 Genetic Analyzer (Applied Biosystems, Thermo Fisher Scientific) and the LightCycler ${ }^{\circledR} 480$ RealTime PCR System (Roche Diagnostics, Basel, Switzerland), respectively.

The WT allele is longer by 11 nucleotides compared to c.18_28delAGAGAGGCCAA allele; this DNA size difference allows the allele separation by capillary

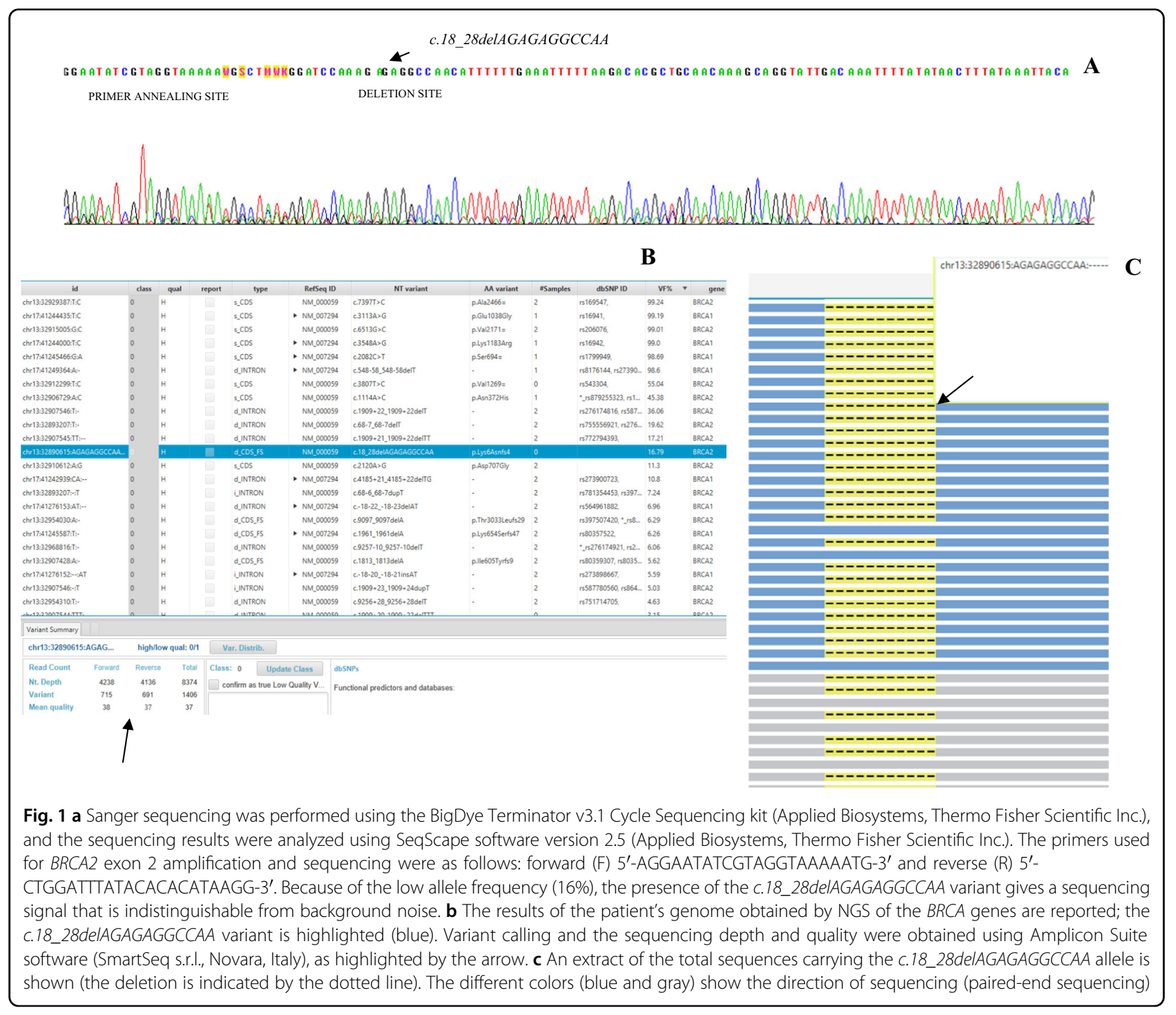




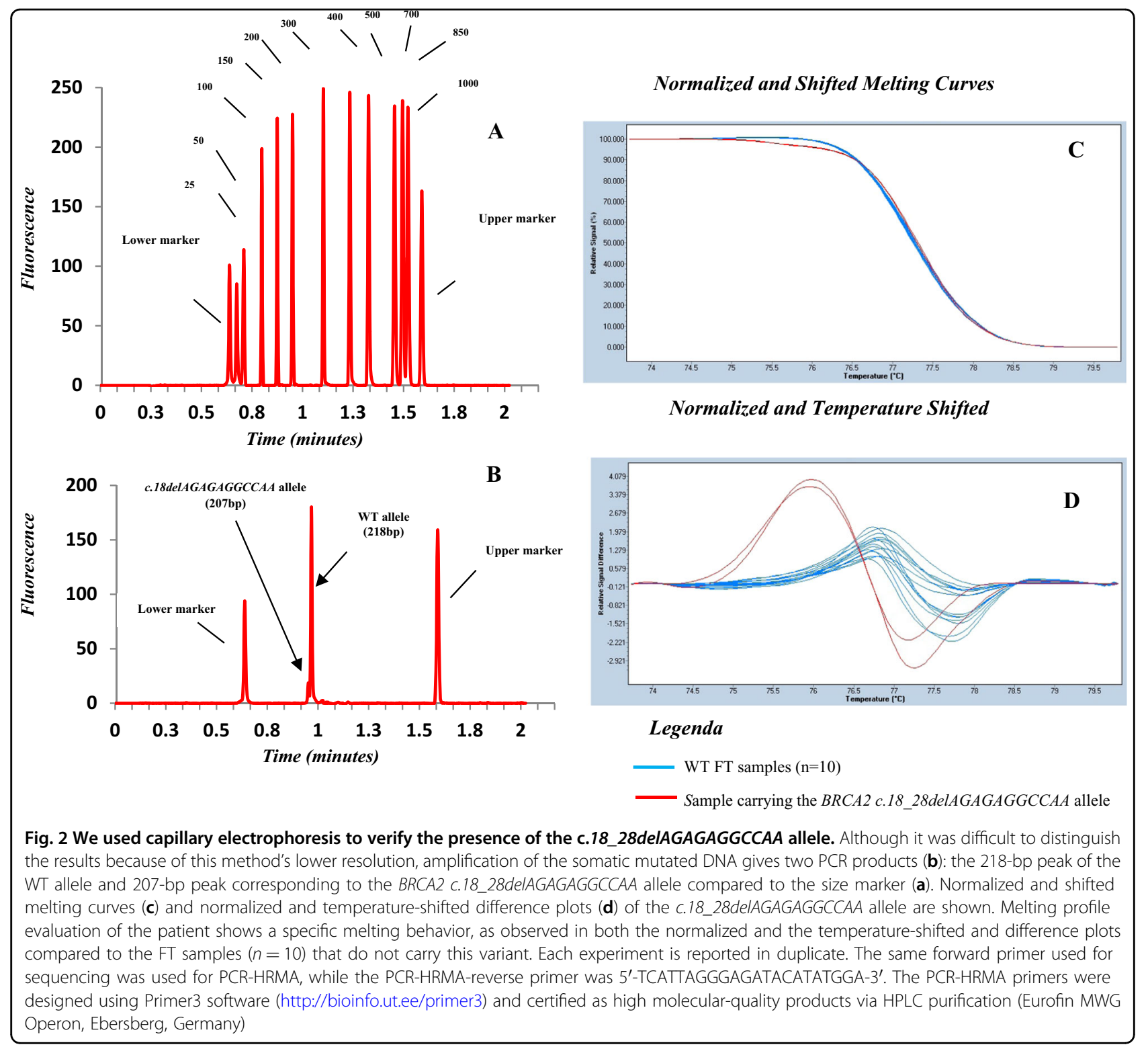

electrophoresis $^{5}$. For this reason, we analyzed the PCR products on an Experion ${ }^{\mathrm{Tx}}$ Automated Electrophoresis System (BioRad, Hercules, CA, USA) following the manufacturer's instructions ${ }^{5}$

$B R C A$ testing obtained by NGS and multiplex ligationdependent probe amplification did not reveal any known pathogenic variants (PVs). However, the patient carried a c.18_28delAGAGAGGCCAA variant in exon 2 of the $B R C A 2$ gene. The nomenclature of the variant is based on the $B R C A 2$ cDNA sequence (NCBI Reference Sequence: NM_000059.3; GRCh37) according to the recommendations of the Human Genome Variation Society (HGVS, http://www.hgvs.org/). The average NGS read depth for the sample was $\sim 10,000 \times$, with a minimum and maximum depth of $2600 \times$ and $32,400 \times$, respectively. The
c.18_28delAGAGAGGCCAA allele showed a read depth of $\sim 1400 \times$ on a total read count of 8700 , resulting in a VAF of $16 \%$.

Sanger sequencing, which was used to confirm the presence of the c.18_28delAGAGAGGCCAA allele, did not reveal this allele (Fig. 1). By contrast, high-resolution melting profiles for the patient showed a specific melting behavior compared to the FT samples $(n=10)$ that did not carry the c.18_28delAGAGAGGCCAA allele.

Finally, capillary electrophoresis also confirmed the presence of the c.18_28delAGAGAGGCCAA allele, although it was difficult to discriminate between the two alleles (Fig. 2).

The c.18_28delAGAGAGGCCAA variant was considered to be a novel variant because it was absent from 
300 FT samples as well as two main variant databases: Clin Var (https://www.ncbi.nlm.nih.gov/clinvar/) and Cosmic (http://cancer.sanger.ac.uk/cosmic). This allele was also considered to be pathogenic because of its deleterious impact on the BRCA2 protein sequence.

With the implementation of treatment-focused $B R C A$ testing on patients with somatic $B R C A \mathrm{PVs}^{6}$, there is an increasing clinical need for routine $B R C A$ screening on DNA from FFPE and FT tumor samples. In this context, it is recommended to perform cost-effective, complete, and accurate $B R C A$ gene sequencing with a sensitivity, throughput, and sample input that cannot be achieved by Sanger sequencing. In fact, Sanger approaches are not fit for detecting low VAFs, leading researchers to confuse these variants with PCR artefacts, which are indistinguishable from the background sequencing noise, and thus consider them false.

For these reasons, many diagnostic laboratories have adopted NGS technology, which offers the potential for fast, cost-efficient, and comprehensive sequencing-based testing of tumor tissue, enabling the identification of somatic $B R C A$ variants.

HRMA represents a high-throughput, rapid, and inexpensive screening test for germline variants ${ }^{7,8}$, and because of its high sensitivity, it has also proven to be effective at identifying somatic variants ${ }^{9}$.

The aim of this study was to report a combined approach with NGS and HRMA to identify and confirm a novel somatic $B R C A 2$ variant. NGS data demonstrated a VAF of $16 \%$, and the depth and quality of the sequencing led us to suspect that this variant was true. Using HRMA as a confirmatory test allowed us to draw up the final clinical report for this patient.

We suggest that this integrated approach can be used in diagnostic settings to improve the molecular assessment of the somatic $B R C A$ status. In fact, our study provides details regarding a rapid and reliable confirmatory assay for low allele-frequency somatic $B R C A$ variants that would otherwise be difficult to confirm with other molecular methods.

Best-practice guidelines for the analysis of FFPE and FT samples ${ }^{10}$ recommend that significant NGS findings must be replicated to ensure the reliability of the results before adopting therapeutic decisions in a clinical context.

This study demonstrates that a combined approach with NGS and HRMA allows for the reliable detection of somatic alterations affecting $B R C A$ genes in FT samples, improving clinical decision-making for the treatment of HGSOC patients.

Finally, epithelial ovarian cancer treatment, which has historically been based on surgery and platinum doublet chemotherapy, is associated with a high risk of relapse and a poor prognosis for recurrent disease. In this landscape, molecular diagnosis of this somatic pathogenic $B R C A 2$ variant made our patient eligible for therapeutic treatments based on poly ADP ribose polymerase, which is a valuable option with promising activity in recurrent ovarian cancer patients and at the different stages of this disease.

\section{HGV Database}

The relevant data from this Data Report are hosted at the Human Genome Variation Database at https://doi. org/10.6084/m9.figshare.hgv.1946

\section{Acknowledgements \\ This study was sponsored by AstraZeneca Pharmaceuticals.}

\section{Author details}

${ }^{1}$ Area Diagnostica di Laboratorio IRCCS, Rome, Italy. ${ }^{2}$ Department of Obstetrics and Gynecology, Division of Gynecologic Oncology, Rome, Italy. ${ }^{3}$ Institute of Biochemistry and Clinical Biochemistry, Teaching and Research Hospital "Agostino Gemelli" Foundation, Rome, Italy. ${ }^{4}$ Proteomics and Metabolomics Unit, IRCCS-Santa Lucia Foundation, Rome, Italy. ${ }^{5}$ Laboratory of Clinical Pathology and Advanced Molecular Diagnostics, Istituto Dermopatico dell'Immacolata -IRCCS, Rome, Italy

Conflict of interest

The authors declare that they have no conflict of interest.

\section{Publisher's note}

Springer Nature remains neutral with regard to jurisdictional claims in published maps and institutional affiliations.

Received: 27 February 2018 Revised: 13 March 2018 Accepted: 30 March 2018.

Published online: 8 June 2018

\section{References}

1. Nik-Zainal, S. et al. Breast Cancer Working Group of the International Cancer Genome Consortium: the life history of 21 breast cancers. Cell 149, 994-1007 (2012).

2. Sims, D., Sudbery, I., llott, N. E., Hegar, A. \& Ponting, C. P. Sequencing depth and coverage: key considerations in genomic analyses. Nat. Rev. Genet. 15, 121-132 (2014).

3. Minucci, A. et al. Clinical impact on ovarian cancer patients of massive parallel sequencing for BRCA mutation detection: the experience at Gemelli hospital and a literature review. Expert Rev. Mol. Diagn. 15, 1383-1403 (2015).

4. Concolino, P. et al. Advanced tools for BRCA1/2 mutational screening: comparison between two methods for large genomic rearrangements (LGRS) detection. Clin. Chem. Lab. Med. 52, 1119-1127 (2014).

5. Minucci, A. et al. Identification of RFLP G6PD mutations by using microcapillary electrophoretic chips (Experion). J. Sep. Sci. 31, 2694-2700 (2008).

6. Kaufman, B. et al. Olaparib monotherapy in patients with advanced cancer and a germline BRCA1/2 mutation. J. Clin. Oncol. 33, 244-245 (2015).

7. Minucci, A., Concolino, P., Giardina, B., Zuppi, C. \& Capoluongo, E. Rapid UGT1A1 (TA)(n) genotyping by high resolution melting curve analysis for Gilbert's syndrome diagnosis. Clin. Chim. Acta 411, 246-249 (2010).

8. Minucci, $A$. et al. High resolution melting analysis is very useful to identify BRCA1 c.4964_4982del19 (rs80359876) founder calabrian pathogenic variant on peripheral blood and buccal swab DNA. Mol. Diagn. Ther. 21, 217-223 (2017).

9. Karbalaie Niya, M. H. et al. Sensitive high-resolution melting analysis for screening of KRAS and BRAF mutations in iranian human metastatic colorectal cancers. Asian Pac. J. Cancer Prev. 17, 5147-5152 (2016).

10. Cree, I. A. et al. Guidance for laboratories performing molecular pathology for cancer patients. J. Clin. Pathol. 67, 923-931 (2014). 